\title{
The Existence of Non-trivial Asymptotically Flat Initial Data for Vacuum Spacetimes
}

\author{
Murray Cantor* \\ Department of Mathematics, Duke University, Durham, NC 27706, USA
}

\begin{abstract}
This paper demonstrates the existence of non-trivial solutions $(g, k)$ to the constraint equations of the initial value formulation of the Einstein field equations over $\mathbb{R}^{3}$ with $g_{i j}-\delta_{i j} \sim|x|^{-1}$ as $|x| \rightarrow \infty$. Using the conformal methods of Lichnerowicz and York, this problem is divided into two parts. First, using weighted Sobolev spaces it is shown the set of pairs $(g, k)$ with $g$ a conformal metric and $k$ transverse-traceless with respect to $g$ forms a smooth vector bundle $\mathscr{P}$ with infinite dimensional fiber. Second, it is shown that the elements of a large open set in $\mathscr{P}$ uniquely determine a solution to the scalar constraint equation with the appropriate growth at infinity, and thereby determine solution to the constraint equations.
\end{abstract}

\section{Introduction}

In writing the Einstein field equations for a vacuum space-time as an evolution system on a 3-manifold $M$, one finds the Cauchy data consists of a Riemannian metric $g_{i j}$ and a symmetric covariant 2-tensor $\pi_{a b}$ satisfying the constraint equations (see Marsden [15]):

$$
\left\{\begin{array}{l}
\operatorname{div}_{g} \pi=0 \\
\pi \cdot \pi-\frac{1}{2}\left(\operatorname{tr}_{g} \pi\right)-R(g)=0 .
\end{array}\right.
$$

Our notation using the summation notation is:

$$
\operatorname{div}_{g} \pi=\pi_{\mid b}^{a b}, \quad \pi \cdot \pi=\pi^{a b} \pi_{a b}, \quad \operatorname{tr}_{g} \pi=g^{a b} \pi_{a b}
$$

and $R(g)$ is the scalar curvature of $g$. Of course covariant differentiation is done with respect to $g$. If $M$ is thought of as a spacelike hypersurface embedded in a spacetime $V^{4}$, and $g_{a b}$ and $k_{a b}$ is the induced metric and second fundamental form of the embedding, then $\pi_{a b}=\left(\left(\operatorname{tr}_{g} k\right) g_{a b}-k_{a b}\right)$. (Note, we will use tensors and not densities.)

The constraint equations form a coupled non-linear system of partial differential equations. The existence of solutions to this system has received much attention

* Current address : Department of Mathematics, The University of Texas, Austin, Texas 78712, USA 
(see for example Choquet-Bruhat [8] for the compact case). A simplification of the problem is due to Lichnerowicz [14] and York [20]. If we assume $\operatorname{tr}_{g} \pi=2 \operatorname{tr}_{g} k=C_{0}$, $C_{0}$ constant on $M$, we may solve (1) by finding a symmetric 2-tensor $\sigma_{T T}$ and a scalar function $\phi$ satisfying the partially uncoupled system:

$$
\left\{\begin{array}{l}
\operatorname{tr}_{g} \sigma_{T T}=\operatorname{div}_{g} \sigma_{T T}=0 \\
8 \Delta_{g} \phi-R \phi+M \phi^{-7}-\frac{1}{6} C_{0} \phi^{5}=0 \\
\phi>0,
\end{array}\right.
$$

where $g$ is any three metric on $M, \Delta_{g} \phi=g^{a b} \phi_{\mid a b}, M=\sigma_{T T}^{i j} \sigma_{T T^{i j}}$. We call $\sigma_{T T}$ a transverse-traceless tensor. A solution of (1) is constructed by setting $\bar{g}_{a b}=\phi^{4} g_{a b}$ $\bar{\pi}=\left(\phi^{-2} \sigma_{T T}\right)+\frac{1}{2} C_{0} \bar{g}$. Furthermore the pair $(\bar{g}, \bar{\pi})$ depends only of the conformal equivalence class of $g$.

For a given $g$ and trace-free $\sigma_{T}, \sigma_{T T}$ may be found by solving:

$$
\operatorname{div}_{g} \sigma_{T}=\left(\operatorname{div}_{g} L_{g}\right)(W),
$$

where $L_{g}$ is the conformal killing operator (see Section 3). If a vector field $W$ solves (2a) then we may set $\sigma_{T T}=\sigma_{T}-L_{g}(W)$. (2a) is often called the vector equation. The second equation in (2) is called the scalar equation.

Note that $\operatorname{tr}_{g} \pi$ depends on the external curvature of the embedding of $M$ in $V^{(4)}$ (in fact it is half the mean curvature) and thus the assumption $\operatorname{tr}_{g} \pi=C_{0}$ amounts to assuming the existence of certain special embeddings of $M$ in a spacetime $V^{4}$. In particular, if $C_{0}=0$ then we must assume there is a "maximal" embedding; i.e. a critical point of the volume operator on the embeddings of $M$ in $V^{(4)}$. The existence of such embeddings has been studied by several authors (Choquet-Bruhat [9], York [20], Cantor et al. [5]).

In the case $M=\mathbb{R}^{3}$, physical considerations leads one to specify that the metric $g$ found in (1) should be asymptotically flat; i.e. it $g_{i j}-\delta_{i j}=0\left(|x|^{-1}\right)$ as $|x| \rightarrow \infty\left(\delta_{i j}=1\right.$ if $i=j$ and $\delta_{i j}=0$ if $i \neq j$ ). It has been shown (Cantor et al. [5]) that a large class of space-times with asymptotically flat space-like hypersurfaces may be foliated by hypersurfaces with $\operatorname{tr}_{g} \pi=\operatorname{tr}_{g} k=0$. Thus, in finding initial data sets for such spacetimes we will consider the system:

$$
\begin{aligned}
& \operatorname{tr}_{g} k=\operatorname{div}_{g} k=0 \\
& 8 \Delta_{g} \phi-R \phi+M \phi^{-7}=0 \\
& \phi>0 .
\end{aligned}
$$

If $(g, k)$ satisfies (3) then $\left(\phi^{4} g,-\phi^{-2} k\right.$ ) satisfies (1). If one interprets $k$ to be $\dot{g}(0)$, the initial velocity of $g(\lambda)$ in the evolution system, one would expect $k_{i j}=0\left(|x|^{-1}\right)$. (Similarly, one may interpret $\pi^{a b}(\operatorname{det} g)^{1 / 2}$ to be the canonical momentum of the system.) However, the theory of elliptic operators (Section 2) requires that the appropriate space for finding transverse-traceless 2-tensors consists of tensors with $|x|^{-2}$ growth at infinity.

These informal observations are made precise in Section 2. This section contains definitions and important properties of the weighted Sobolev spaces $M_{s, \delta}^{p}$. These spaces were first considered by Nirenberg and Walker [14] and Cantor [2-4]. They have proven to be useful in studying asymptotically homogeneous elliptic operators on $\mathbb{R}^{n}$. 
In Section 3 the nature of the space $\mathscr{P}$ of pairs $(g, k)$ where $g$ is a asymptotically flat conformal metric $\left(\operatorname{det} g_{i j}=1\right)$ and $\operatorname{div}_{g} k=\operatorname{tr}_{g} k=0$ is studied. It is shown that with the appropriate topology $\mathscr{P}$ is a fiber bundle over the space of conformal metrics. Furthermore, the fibers of this bundle are infinite dimensional. Also in this section we obtain a precise version of York's decomposition of symmetric 2-tensors.

In Section 4 the scalar equation is considered. It is shown that each $(g, k)$ in a large open set of $P$ uniquely determines a solution $\phi$ of the scalar equation. Furthermore the solution $\phi$ depends smoothly on $(g, k)$.

A new result in this section (Theorem 4.2) is that for an asymptotically flat metric $g$ with scalar curvature $R \leqq 0$ and function $\bar{R}$ with $C^{2} \bar{R} \leqq R \leqq \bar{R} \leqq 0$ there is a asymptotically flat metric $\bar{g}$ such that $\bar{g}$ is conformally equivalent to $g$ and the scalar curvature of $\bar{g}$ is conformally equivalent to $g$ and the scalar curvature of $\bar{g}$ is $\bar{R}$. A discussion of problems of this sort may be found in Kazden and Warner [12].

The author wishes to thank Professor James W. York for his patience and encouragement during our many discussions on this topic.

\section{Mathematical Tools}

Many of the results of this paper depend on finding solutions to elliptic systems with specified asymptotic behavior at infinity. In this context the following spaces of functions have proven to be useful:

Definition 2.1. Let ||$_{p}$ be the standard $L^{p}$ norm, and $\sigma(x)=\left(1+|x|^{2}\right)^{1 / 2}$. For $s \in \mathbb{N}$, and $\delta \in \mathbb{R}$ and $f: \mathbb{R}^{n} \rightarrow \mathbb{R}^{m}$ set

$$
|f|_{p, s, \delta}=\sum_{|\alpha| \leqq s}\left|\sigma^{\delta+|\alpha|} D^{\alpha} f\right|_{p}
$$

Note that $\|_{p, s, \delta}$ is a norm on $C_{0}^{\infty}\left(\mathbb{R}^{n,} \mathbb{R}^{m}\right)$ and if $\delta \geqq 0$ is stronger than the usual Sobolev norms. We use these norms to construct certain Banach spaces.

Definition 2.2. Denote by $M_{s, \delta}^{p}\left(\mathbb{R}^{n,} \mathbb{R}^{m}\right)$ the completion of $C_{0}^{\infty}\left(\mathbb{R}^{n}, \mathbb{R}^{m}\right)$ with respect to $\|_{p, s, \delta}$

Usually, when there is no chance of confusion we shall just use $M_{s, \delta}^{p}$.

We shall have need of the following lemmas whose proofs appear elsewhere:

Lemma 2.3. If $p \geqq 1$, and $s>n / p+k$ then $M_{s, \delta}^{p} \subset C^{k}$ continuously.

Proof. This follows a fortiori from the standard Sobolev inequalities.

Lemma 2.4. Let $p>1, s>(n / p), \delta \geqq 0$ and $0 \leqq l \leqq s$ then pointwise multiplication induces a continuous (and hence smooth) map

$$
M_{s, \delta}^{p} \oplus M_{s-l, \delta+l}^{p} \rightarrow M_{s-l ; \delta+l}^{p} .
$$

For a proof see Cantor [2], Proposition 1.1.

Lemma 2.5. Let $M_{s, \delta}^{p}(1)=\left\{g: \mathbb{R}^{3} \rightarrow \mathbb{R}: g-1 \in M_{s, \delta}^{p}\right\}$ be given the topology such that the map $g \rightarrow g+1$ from $M_{s, \delta}^{p}$ is continuous. Then for $p>1, s>(n / p)$ and $\delta \geqq 0$ pointwise 
multiplication induces smooth maps:

$$
\begin{aligned}
& M_{s, \delta}^{p}(1) \times M_{s-l, \delta+l}^{p}(1) \rightarrow M_{s-l, \delta+l}^{p}(1) \\
& M_{s, \delta}^{p}(1) \times M_{s-l, \delta+l}^{p} \rightarrow M_{s-l, \delta+l}^{p} .
\end{aligned}
$$

Proof. Suppose $f, g \in M_{s, \delta}^{p}(1)$. Then writing $f=\bar{f}+1$ and $g=\bar{g}+1$ with $\bar{f}, \bar{g} \in M_{s, \delta}^{p}$, we get

$$
f \cdot g-1=\bar{f} \cdot \bar{g}+\bar{f}+\bar{g} .
$$

Which by Lemma 2.3 is in $M_{s, \delta}^{p}$ and the function is clearly continuous. Q.E.D.

Lemma 2.6. Let $p>1, s>n / p$ and $\delta \geqq 0$ and $U=\left\{f: \mathbb{R}^{n} \rightarrow \mathbb{R}: f-1 \in M_{s, \delta}^{p}\right.$ and $f(x)$ $>0\}$. Then $f \rightarrow(f)^{\alpha}$ is a smooth function on $U$, for any $\alpha \in \mathbb{R}$ (see Cantor [2]).

Lemma 2.7. Let $g_{a b}$ be a $C^{1}$ bounded Riemannian metric on $\mathbb{R}^{3}$ with $\lim _{|x| \rightarrow \infty}\left|g_{i j}(x)-\delta_{i j}\right|$ $=0, \Delta_{g} \phi=\sum_{a, b} g^{a b} \phi_{\mid a b}$ (covariant differentiation with respect to $g$ ). Then if $f$ is $a$ continuous positive function on $\mathbb{R}^{3}$ and $\Delta_{g} \phi-f \cdot \phi \geqq 0$ with $\lim _{|x| \rightarrow \infty} \phi(x)=0$ then $\phi \leqq 0$.

Proof. In the coordinate expression for $\Delta_{g}$ we see it is a uniformly elliptic operator with no zeroth order terms. Thus we may apply Theorem 6, Chapter 2 of Protter and Weinberger [19] to conclude if there is a $p \in \mathbb{R}^{3}$ where $\phi$ takes its maximum and $\phi(p)>0$ then $\phi$ is constant. This easily leads to a contradiction. Q.E.D.

We will require both of the following theorems.

Theorem 2.8. Let $p>n /(n-2), s>n / p+20 \leqq \delta<-2+n(p-1) / p$ and $A_{\infty}=\sum_{|\alpha|=2} \bar{a}_{\alpha} D^{\alpha}$ a second order homogeneous elliptic operator with constant coefficients. If $A(x)$ $=\sum_{|\alpha| \leqq 2} a_{\alpha}(x) D^{\alpha}$ is an elliptic operator on $\mathscr{M}_{s, \delta}^{p}$ such that

$$
\begin{array}{ll}
a_{\alpha} \in \mathscr{M}_{s-2,2-|\alpha|}^{p} & |\alpha|<2 \\
\left(a_{\alpha}-\bar{a}_{\alpha}\right) \in \mathscr{M}_{s-2,0}^{p} & |\alpha|=2 .
\end{array}
$$

Then $A: \mathscr{M}_{s, \delta}^{p} \rightarrow \mathscr{M}_{s-2, \delta+2}^{p}$ is a continuous map with closed range and finite dimensional kernal.

Furthermore, suppose one of the following conditions hold:

i) $\sum_{|\alpha|<2}\left|\alpha_{\alpha}\right|_{p, s-2,2-|\alpha|}+\sum_{|\alpha|=2}\left|a_{\alpha}-\bar{a}_{\alpha}\right|_{p, s-2,0}<\varepsilon$ for sufficiently small positive $\varepsilon$.

ii) There is a continuous curve con $[0,1]$ into the space of bounded linear operators between $\mathscr{M}_{s, \delta}^{p}$ and $\mathscr{M}_{s-2, \delta+2}^{p}$ such that $c(0)=A_{\infty}, c(1)=A$ and $c(t)$ is an injection for all $t \in[0,1]$.

Then $A$ is an isomorphism of $\mathscr{M}_{s, \delta}^{p}$ and $\mathscr{M}_{s-2, \delta+2}^{p}$.

For a proof see Cantor [3], Theorem 1.4. 
Theorem 2.9. Let $p, \delta$, and $A_{\infty}$ be as in Theorem 2.8. Let $k \geqq 2 A(x)=\sum_{|\alpha| \leqq 2} a_{\alpha}(x) \mathscr{D}^{\alpha}$ be an elliptic operator such that $a_{\alpha} \in C^{k-2}$ and for each multi-index $\gamma, 0 \leqq|\gamma| \leqq k-2$

$$
\begin{aligned}
& \limsup _{|x| \rightarrow \infty}\left|\left(\mathscr{D}^{\gamma} a_{\alpha}(x)\right) \sigma(x)^{m-|\alpha|+|\gamma|}\right|<\varepsilon \quad|\alpha|<2 \\
& \limsup _{|x| \rightarrow \infty}\left|\mathscr{D}^{\gamma}\left(a_{\alpha}(x)-\bar{a}_{\alpha}\right) \sigma^{|\gamma|}(x)\right|<\varepsilon \quad \text { for } \quad|\alpha|=2
\end{aligned}
$$

for sufficiently small $\varepsilon>0$. Then $A$ maps $\mathscr{M}_{k, \delta}^{p}$ into $\mathscr{M}_{k-2, \delta+2}^{p}$ continuously with closed range and finite dimensional kernel. Furthermore if (i) of Theorem 2.8. holds then $A$ is an isomorphism.

This is proven in Cantor [3], Theorem 1.3.

We would like to make several remarks concerning the use of these theorems.

1. Nirenberg and Walker [16] have shown the given ranges of $p$ and $\delta$ are essential for the theorems to hold. Thus we may use these inequalities to study typical growth at infinity of solutions to homogeneous equations.

2 . For $n=3$, note that $p>3$ and the range of $\delta$ is such that functions of growth $|x|^{-1}$ are included [actually slightly faster growth such as $\ln (|\mathrm{x}|) /|\mathrm{x}|$ is allowed]. Thus if $A_{\infty} f=g \in C_{0}^{\infty}$ then heuristically one may expect $f$ to have $|x|^{-1}$ growth at infinity.

3. Derivatives of functions in $M_{s, \delta}^{p}$ fall successively faster at infinity. Thus if $n=3$ and $A_{\infty} f \in C_{0}^{\infty}$ then if $|\alpha|=1, D^{\alpha} f$ would fall like $|x|^{-2}$ at infinity.

\section{Conformal Metrics and York's Decomposition}

In this section we will construct a candidate for the parameterization space $\mathscr{P}$ of initial data. This space will consist of pairs $(g, k)$ with $\operatorname{div}_{g} k=\operatorname{tr}_{g} k=0$. Of course these pairs satisfy two of the three constraint equations. The topology will be chosen to be both physically reasonable and to give $\mathscr{P}$ the structure of a vector bundle.

In the following section we will show that members of a large open set in $\mathscr{P}$ do uniquely and smoothly specify solutions to the initial data equations.

Definition 3.1. Let $\gamma=\delta_{i j}$ be the Euclidean metric on $\mathbb{R}^{3}$ and let $\mathscr{R}_{s, \delta}^{p}=\{$ Riemannian metrics $g$ on $\mathbb{R}^{3}$ such that $\left.g-\gamma \in \mathscr{M}_{s, \delta}^{p}\right\}$. Also let $S_{s, \delta}^{p}=\left\{\right.$ covariant 2-tensors in $\left.\mathscr{M}_{s, \delta}^{p}\right\}$.

Note if $s>3 / p$ since the positive definite property is open in $C^{0}, \mathscr{R}_{s, \delta}^{p}$ is open in $S_{s, \delta}^{p}$ $+\{\gamma\}$ and therefore has a natural manifold structure. Also it follows for $g \in \mathscr{R}_{s, \delta}^{p}$ the tangent space $T_{g} \mathscr{R}_{s, \delta}^{p} \simeq S_{s, \delta}^{p}$.

Let $d V=d x^{1} \wedge d x^{2} \wedge d x^{3}$ be the volume form associated with $\gamma$. If $g$ is any metric on $\mathbb{R}^{3}$, it is well known there is a relative scalar function $|g|$ such that the volume form $\mu_{g}$ associated with $g$ may be written as $\mu_{g}=|g| d V$. In fact, $|g|=\left(\operatorname{det} g_{i j}\right)^{1 / 2}$.

Definition 3.2. Let $\mathscr{C}_{s, \delta}^{p}=\left\{g \in \mathscr{R}_{s, \delta}^{p}:|g|=1\right\}$.

It is easy to check that $\mathscr{C}_{s, \delta}^{p}$ represents the set of conformal structures for metrics in $\mathscr{R}_{s, \delta}^{p}$. Every conformal equivalence class in $\mathscr{R}_{s, \delta}^{p}$ has exactly one representative in $\mathscr{C}_{s, \delta}^{p}$. In fact if $g \in \mathscr{R}_{s, \delta}^{p}$ and $\tilde{g}=|g|^{-2 / 3} g$, then $|\tilde{g}|=1$ and $\tilde{g}$ is conformally equivalent to g.

Proposition 3.3. Let $p>1, s>3 / p+1$, and $\delta \geqq 0$. If $g \in \mathscr{R}_{s, \delta}^{p}$ then $|g|^{-2 / 3} g \in \mathscr{C}_{s, \delta}^{p}$. 
Proof. This is an immediate application of the product and composition theorems found in Section 2.

Proposition 3.4. Let $p>1, s>3 / p+1, t \leqq s, \delta \geqq 0$ and $\delta^{\prime} \geqq 0$. Then if $g \in \mathscr{R}_{s, \delta}^{p}$

$$
S_{t, \delta^{\prime}}^{p}=\operatorname{tr}_{g}\left(S_{t, \delta^{\prime}}^{p}\right) g \oplus\left\{h \in S_{t, \delta^{\prime}}^{p}: \operatorname{tr}_{g} h=0\right\} \text {. }
$$

Proof. We call $E_{t}=\left\{h \in S_{t, \delta^{\prime}}^{p}: \operatorname{tr}_{g} h=0\right\}$.

Let $g_{i j}=\delta_{i j}+h_{i j}$ with $h_{i j} \in \mathscr{M}_{s, \delta}^{p}$. Also write $g^{i j}=\delta^{i j}+\bar{h}^{i j}$. It follows from Cramers formula that $\bar{h}^{i j} \in \mathscr{M}_{s, \delta}^{p}$. Now for $X \in S_{t, \delta^{\prime}}^{p}$

$$
\begin{aligned}
\left(\operatorname{tr}_{g} X\right) g_{k l} & =\left[\left(\delta^{i j}+\bar{h}^{i j}\right) X_{i j}\right]\left(\delta_{k l}+h_{k l}\right) \\
& =\left(X_{i}^{i}+\bar{h}^{i j} X_{i j}\right) \delta_{k l}+\left(X_{i}^{i}+\bar{h}^{i j} X_{i j}\right) h_{k l} .
\end{aligned}
$$

Now $X_{i}^{i} \in \mathscr{M}_{t, \delta^{\prime}}^{p}, \bar{h}^{i j} X_{i j} \in \mathscr{M}_{t, \delta+\delta^{\prime}}^{p} \mathscr{M}_{t, \delta^{\prime}}^{p}$ and $\left(X_{i}^{i}+\bar{h}^{i j} X_{i j}\right) h_{k l} \in \mathscr{M}_{t, \delta+\delta^{\prime}}^{p}$

Thus we may write

$$
X=\frac{1}{3}\left(\operatorname{tr}_{g} X\right) g+\left(X-\frac{1}{3} \operatorname{tr}_{g} X g\right)
$$

and conclude

$$
S_{t, \delta^{\prime}}^{p}=\operatorname{tr}_{g}\left(S_{t, \delta^{\prime}}^{p}\right)+E_{t},
$$

where both summands are subspaces of $S_{t, \delta^{\prime}}^{p}$. It is easily seen that $\operatorname{tr}_{g}\left(S_{s, \delta^{\prime}}^{p}\right) \cap \operatorname{Ker}\left(\operatorname{tr}_{g}\right)$ $=\{0\}$ and the summands are closed.

Theorem 3.5. Let $p>1, s>3 / p+1$ and $\delta>0$. Then $\mathscr{C}_{s, \delta}^{p}$ is a smooth submanifold of $\mathscr{R}_{s, \delta}^{p}$. Also for $g \in \mathscr{C}_{s, \delta}^{p}$

$$
T_{g} \mathscr{C}_{s, \delta}^{p}=\left\{V \in S_{s, \delta}^{p}: \operatorname{tr}_{g} V=0\right\} .
$$

Proof. Let $\Phi: \mathscr{R}_{s, \delta}^{p} \rightarrow \mathscr{M}_{s, \delta}^{p}(1)$ (see Lemma 2.5)

$$
\Phi(g)=\operatorname{det}\left(g_{i j}\right) .
$$

Note $\mathscr{C}_{s, \delta}^{p}=\Phi^{-1}(1)$ and it follows from Lemma 2.5 that $\Phi$ is $C^{\infty}$. We need show $\Phi$ is a submersion. Applying the classical formula

$$
\left.\frac{d}{d t} \operatorname{det}\left(A_{t}\right)\right|_{t=0}=\operatorname{trace}\left(\left.A_{0}^{-1}\left(\frac{d}{d z} A_{t}\right)\right|_{t=0}\right)
$$

we see $D \Phi(g)(V)=\operatorname{tr}_{g} V$. This is clearly a surjection from $S_{s, \delta}^{p}$ to $\mathscr{M}_{s, \delta}^{p}$ and by Proposition 3.4 (setting $\delta^{\prime}=\delta$ ) it has a splitting kernal. This result follows then from standard manifold theory (see Lang [13]). Q.E.D.

Definition 3.6. Let $X_{s, \delta}^{p}$ be the vector fields on $\mathbb{R}^{3}$ in $\mathscr{M}_{s, \delta}^{p}$. Let $X$ be vector field on $\mathbb{R}^{3}$ and $L_{X}$ represent Lie differentiation with respect to $X$. Then if $g$ is a metric on $\mathbb{R}^{3}$ we have the conformal killing operator $L_{g}: X_{s, \delta}^{p} \rightarrow S_{s-1, \delta+1}^{p} L_{g}(X)=L_{X} g-\frac{2}{3} \operatorname{div}_{g} X$.

Theorem 3.7 (York's Decomposition). Let $p>3, s>3 / p+2,0 \leqq \delta<-2+3(p-1) / p$. Then for $g \in \mathscr{R}_{s, \delta}^{p}$ and $t=s-1$ or $t=1$

$$
S_{t, \delta+1}^{p}=L_{g}\left(X_{t+1, \delta}^{p}\right) \oplus \operatorname{tr}_{g}\left(S_{t, \delta+1}^{p}\right) g \oplus J_{t},
$$

where $J_{t}=\left\{k \in S_{t, \delta+1}^{p}: \operatorname{tr}_{g} k=\operatorname{div}_{g} k=0\right\}$. Furthermore each $J_{t}$ is infinite dimensional. 
Proof. We first note that Proposition 3.4 allows us to write for $t \leqq 2$

$$
S_{t, \delta+1}^{p}=\operatorname{tr}_{g}\left(S_{t, \delta+1}^{p}\right) \oplus E_{t},
$$

where $E_{t}=\left\{h \in S_{t, \delta+1}^{p}: \operatorname{tr}_{g} h=0\right\}$. Also notice that $L_{g}\left(X_{t+1, \delta}^{p}\right) \subset E_{t}$. Let $L_{t}=L_{g}\left(\mathscr{M}_{t+1, \delta}^{p}\right)$ and $J_{t}=\left\{h \in E_{t}: \operatorname{div}_{g} h=0\right\}$. We claim that for $t=s-1$ or $t=1$

$$
E_{t}=L_{t} \oplus J_{t} .
$$

To show this let $\beta \in L_{t}$ and consider the system:

$$
\operatorname{div}_{g} \circ L_{g}(\alpha)=\operatorname{div}_{g} \beta \text {. }
$$

Following York [21], $A=\operatorname{div}_{g} \circ L_{g}$ is an elliptic operator and since $g \in \mathscr{R}_{s, \delta^{\prime}}^{p}$ the coefficients of $A$ satisfy the conditions of Theorem 2.8. Also by standard Sobolev arguments $A$ satisfies the hypotheses of Theorem 2.9 with $k=2$.

Thus, in either case we have

$$
\operatorname{div}_{g} \circ L_{g}: X_{t+1, \delta}^{p} \rightarrow X_{t-1, \delta+2}^{p}
$$

is an isomorphism. Since $\operatorname{div} \beta \in X_{t-1, \delta+2}$ we have $\alpha \in \mathscr{M}_{t+1, \delta}^{p}$. We may write

$$
\beta=L_{g}(\alpha)+\left(\beta-L_{g}(\alpha)\right) \text {. }
$$

Note $\operatorname{div}_{g}\left(\beta-L_{g}(\alpha)\right)=0$. Thus

$$
E_{t}=L_{t}+J_{t} .
$$

If $\beta=L_{g}(\alpha)$ and $\operatorname{div}_{g}(\beta)=0$ then $\operatorname{div}_{g} L_{g}(\alpha)=0$ which implies $\alpha=0$ and thus $\beta=0$. The summands, $L_{g}\left(\mathscr{M}_{t+1, \delta}^{p}\right)$ and $J_{t}$ are kernals of continuous maps and hence closed.

We shall now show $J_{t}$ is infinite dimensional. We will follow the argument of Bourguignon et al. [1].

Note that $\bigcap_{t \geqq 1} L_{t}$ is dense in $L_{t}$ for all $t \geqq 1$. Let $J=\bigcap_{t \geqq 1} J_{t}$. Since the summands in (4) are closed, we have that $J$ is dense in $J_{t}$. In particular if $J$ were finite dimensional it would follow that $J=J_{1}$. The same argument shows if $J_{s}$ were finite dimensional then $J_{s}=J_{1}$.

Note $\operatorname{div}_{g}: L_{1} \rightarrow X_{0, \delta+2}^{p}$ is an isomorphism and thus there is a constant $C_{1}$ such that for each $h \in L_{1}$,

$$
|h|_{p, 1, \delta+1} \leqq C_{1}\left|\operatorname{div}_{g} h\right|_{p, 0, \delta+2} \text {. }
$$

Let $h \in E_{1}$. We may write $h=h^{\prime}+h^{\prime \prime}$ where $h^{\prime} \in L_{1}$ and $h^{\prime \prime} \in J_{1}$. Then

$$
\begin{aligned}
|h|_{p, 1, \delta+1} & \leqq\left|h^{\prime}\right|_{p, 1, \delta+1}+\left|h^{\prime \prime}\right|_{p, 1, \delta+1} \\
& \leqq C\left(\left|\operatorname{div}_{g} h\right|_{p, 0, \delta+2}+\left|h^{\prime \prime}\right|_{p, 1, \delta+1}\right) .
\end{aligned}
$$

If $J_{1}$ were finite dimensional there would be a constant $C_{2}$ such that

$\left|h^{\prime \prime}\right|_{p, 1, \delta+1} \leqq C_{2}\left|h^{\prime \prime}\right|_{p, 0, \delta+1}$.

Thus we would have

$$
|h|_{p, 1, \delta+1} \leqq C_{3}\left(\left|\operatorname{div}_{g} h\right|_{p, 0, \delta+2}+|h|_{p, 0, \delta+1}\right) \text {. }
$$


Let $h$ be any symmetric 2-tensor in $C_{0}^{\infty}\left(\mathbb{R}^{3}\right)$. For $\xi_{0} \neq 0 \in\left(\mathbb{R}^{3}\right)^{*}$ and $\lambda \in \mathbb{R}$, set $h_{y}$ $=e^{i \lambda\left\langle x, \xi_{0}\right\rangle} h$. Also let $\sigma_{g}$ be the principle symbol of $\operatorname{div}_{g}$. Then we have

$$
\lambda^{-1} e^{-i \lambda\left\langle x, \xi_{0}\right\rangle} \operatorname{div}_{g}\left(h_{y}\right) \rightarrow \sigma_{g}\left(x, \xi_{0}\right) h \quad \text { as } \lambda \rightarrow \infty .
$$

Also, there is constant $C_{4}$ such that

$$
\begin{aligned}
\left|h_{\lambda}\right|_{p, 1, \delta+1} & =\left|h_{\lambda}\right|_{p, 0, \delta+1}+\left|D\left(h_{\lambda}\right)\right|_{p, 0, \delta+2} \\
& =|h|_{p, 0, \delta+1}+\lambda\left|\xi_{0}\right||h|_{p, 0, \delta+2}+|D h|_{p, 0, \delta+2} \\
& \geqq C_{4} \lambda|h|_{p, 0, \delta+1} .
\end{aligned}
$$

Now

$$
\begin{aligned}
|h|_{p, 0, \delta+1} & \leqq C_{4} \lambda^{-1}\left|h_{\lambda}\right|_{p, 1, \delta+1} \\
& \leqq C_{5} \lambda^{-1}\left(\left|\operatorname{div}_{g} h_{\lambda}\right|_{p, 0, \delta+2}+\left|h_{\lambda}\right|_{p, 0, \delta+1}\right) .
\end{aligned}
$$

If $\lambda>1 / C_{5}$ we have

$$
\begin{aligned}
|h|_{p, 0, \delta+1} & \leqq C_{5} \lambda^{-1}\left(\left|\operatorname{div}_{g} h_{\lambda}\right|_{p, 0, \delta+2}\right. \\
& \leqq C_{5}\left|\sigma_{g}\left(\cdot, \xi_{0}\right) h\right|_{p, 0, \delta+2} .
\end{aligned}
$$

It follows for any symmetric 2-tensor $h \in C_{0}^{\infty}\left(\mathbb{R}^{3}\right)$ and $x \in \mathbb{R}^{3}, \xi_{0} \in\left(R^{3}\right)^{*}$ there is a constant $C_{6}$ such that

$$
|h(x)| \leqq C_{6}\left|\sigma_{g}\left(x, \xi_{0}\right)(h(x))\right| .
$$

This implies $\sigma_{g}$ is an injective symbol when restricted to $E_{1}$. However, as pointed out in Proposition 3 of Bourguignon et al. [1], this is impossible. Q.E.D.

Corollary 3.7.1. Under the assumptions of Theorem 3.7 there are an infinite number of linearly independent solutions to $\operatorname{div}_{g} k=\operatorname{tr}_{g} k=0$ with $k \in S_{s-1, \delta+1}^{p}$.

The decomposition in Theorem 3.7 arises naturally when studying the quotient space of $\mathscr{C}_{s, \delta}^{p}$ under the action of the diffeomorphism group (Coordinate Changes). $J_{s}$ represents infinitesimal changes of conformal geometry (see Cantor [3], Fischer and Marsden [11], or York [21] for details).

Theorem 3.8. Let $p>3, s>3 / p+2$, and $0 \leqq \delta<-2+3(p-1) / p$. Then

$$
\left.\mathscr{P}=(g, k) \in \mathscr{C}_{s, \delta}^{p} \times S_{s-1, \delta+1}^{p}: \operatorname{tr}_{g} k=\operatorname{div}_{g} k=0\right\}
$$

is a smooth sub-bundle of $\mathscr{C}_{s, \delta}^{p} \times S_{s-1, \delta+1}^{p}$.

Proof. We apply Proposition 6, Theorem III of Lang [13] which states if $f: \pi \rightarrow \pi^{\prime \prime}$ is a vector bundle morphism of vector bundles over a Banach manifold $X$ such that for each $x \in X, f_{x}: E_{x} \rightarrow E_{x}^{\prime \prime}$ is surjective and has a kernal that splits. Then the sequence $\pi \stackrel{f}{\rightarrow} \pi^{\prime \prime} \rightarrow 0$ is exact.

It follows that $\operatorname{Ker} f=\cup \operatorname{Ker}\left(f_{x}\right)$ is a sub-bundle (see Lang [13], p. 43).

We first consider

$$
\begin{aligned}
\operatorname{tr}: & \mathscr{C}_{s, \delta}^{p} \times S_{s-1, \delta+1}^{p} \rightarrow \mathscr{C}_{s, \delta}^{p} \times S_{s-1, \delta+1}^{p} \\
(g, k) & \mapsto\left(g, \operatorname{tr}_{g} k\right) .
\end{aligned}
$$


It follows from Proposition 3.4 that tr satisfies the conditions of the above proposition so that ker $t_{r}=E$ is a sub-bundle.

We now consider

$$
\begin{gathered}
\operatorname{div}: E \rightarrow \mathscr{C}_{s, \delta}^{p} \times X_{s-2, \delta+2}^{p} \\
(g, h) \rightarrow\left(g, \operatorname{div}_{g} h\right) .
\end{gathered}
$$

This is clearly a vector bundle morphism and it is shown in the proof of Theorem 3.7 that on each fiber $E_{g}, \operatorname{div}_{g}$ is a surjection with a splitting kernal. Thus $\mathscr{P}=\operatorname{ker}(\mathrm{div})$ is a sub-bundle of $E$. The theorem follows immediately. Q.E.D.

\section{The Conformal Factor}

In this section it is shown that members of a large subset of $\mathscr{P}$ uniquely and smoothly determine a solution of the scalar constraint equation. The problem we wish to solve is

$$
\left\{\begin{array}{l}
\Delta_{g} \phi-R(g) \phi+M \phi^{-7}=0 \\
\phi>0 \\
\phi-1 \in M_{s, \delta}^{p},
\end{array}\right.
$$

where $R$ is the Scalar curvature of $g, M=k \cdot k=k^{i j} k_{i j}$ and $p, s, \delta$ are as in Theorem 3.8. Note $\phi-1$ has $|x|^{-1}$ growth at infinity.

The scalar constraint equation has been studied by Choquet-Bruhat [7] who obtained similar results to the $R \geqq 0$ case below. She used her notion of asymptotic Holder spaces and had to assume globally small $C^{0}$ norms for $R$ and $M$. We need the weaker assumption that $R(g)$, and $M$ are in $M_{s-2, \delta+2}^{p}$.

In what follows $\left(\Delta_{g} \phi\right)=g^{a b} \nabla_{a} \nabla_{b} \phi$ and so it is sufficient that $g_{i j}-\delta_{i j} \in M_{s, \delta}^{p}$ in order to apply Theorem 2.8 to $\Delta_{g}$.

Theorem 4.1 (Positive Curvature). Let $p>3,0 \leqq \delta<-2+3(p-1) / p$ and $s>n / p+2$. Let $g \in \mathscr{R}_{s, \delta}^{p}$ have nonnegative scalar curvature $R(g)$ and let $M \in M_{s-2, \delta+2}^{p}\left(\mathbb{R}^{3}, \mathbb{R}\right)$ have the property there exist positive constants $C_{1}$ and $C_{2}$ such that $0 \leqq C_{1} R(x) \leqq M(x)$ $\leqq C_{2} R(x)$ for all $x \in \mathbb{R}^{3}$. Then there is a neighborhood $U$ of $(g, M)$ in $\mathscr{R}_{s, \delta}^{p} \times M_{s-2, \delta+2}^{p}$ such that if $\left(g^{\prime}, M^{\prime}\right) \in U$ there is a unique $\phi$ satisfying:

$$
\begin{gathered}
8 \Delta_{g^{\prime}} \phi-R\left(g^{\prime}\right) \phi+M^{\prime}(\phi)^{-7}=0 \\
\phi-1 \in M_{s, \delta}^{p} \\
\phi(x)>0 \quad \text { for all } x \in \mathbb{R}^{3} .
\end{gathered}
$$

Furthermore, $\phi$ depends smoothly on $\left(g^{\prime}, M^{\prime}\right)$ in $U$.

Proof. We prove this in several steps. First using a monotone convergence scheme we solve the equation using the given $g$ and $M$. We then complete the theorem using the implicit function theorem. Throughout this proof let $R=R(g)$. Note $R \in M_{s-2, \delta+2}^{p}$. 
Let $\phi=1+u$ we substitute into $\left(\mathrm{C}^{\prime}\right)$ to obtain the following reformulation:

$$
\left\{\begin{array}{l}
8 \Delta_{g} u-R u+M(1+u)^{-7}=R \\
u \in M_{s, \delta}^{p} \\
u(x)>-1 \text { for all } x \in \mathbb{R}^{3} .
\end{array}\right.
$$

Set $f(u)=8^{-1}\left(R(u+1)-M(u+1)^{-7}\right)$. Note using Lemmas 2.4 and 2.5 we see $f: M_{s, \delta}^{p} \rightarrow M_{s-2, \delta+2}^{p}$ smoothly. Also $\frac{\partial f}{\partial u}(x)=8^{-1}\left(R(x)+7 M(x)(u(x)+1)^{-8} \geqq 0\right.$ when $u(x)>-1$. Thus $f$ is increasing in $u$ for the functions under consideration.

Step 1. Uniqueness.

Let $v_{1}$ and $v_{2}$ be two solutions of $\left(\mathrm{C}^{\prime \prime}\right)$. Then $w=v_{1}-v_{2}$ is continuous. Suppose $w(x) \neq 0$. Then we may assume without loss of generality that there is a set $D \subset \mathbb{R}^{3}$ such that $w>0$ on $D$. But on $D, \Delta_{g} w=f\left(v_{1}\right)-f\left(v_{2}\right) \geqq 0$ and therefore $w$ cannot take a maximum on $D$. Thus $\bar{D}$ is contained in the interior of a larger set with $w>0$. In fact, it follows $w \geqq 0$ on $\mathbb{R}^{3}$ and $\Delta_{g} w \geqq 0$ on $\mathbb{R}^{3}$. Then using Lemma 2.7 , we see $w$ must vanish everywhere.

Step 2. Monotone convergence.

We first establish the following: There exist constants $e_{1}>-1$ and $e_{2}>e_{1}$ such that for all $x \in \mathbb{R}^{3}$,

$$
\begin{aligned}
& 0 \geqq R(x)\left(1+e_{1}\right)-M(x)\left(1+e_{1}\right)^{-7} \\
& 0 \leqq R(x)\left(1+e_{2}\right)-M(x)\left(1+e_{2}\right)^{-7} .
\end{aligned}
$$

Proof. Let $C_{1}=\left(1+e_{1}\right)^{8}$ and $C_{2}=\left(1+e_{2}\right)^{8}$ then clearly $e_{2}>e_{1}$ and dividing the inequality

$$
\left(1+e_{1}\right)^{8} R(x) \leqq M(x)
$$

by $\left(1+e_{1}\right)^{7}$ yields the first inequality. The second inequality is obtained similarly.

Given $R, M, e_{1}$ and $e_{2}$ let $u_{1} \in M_{s, \delta}^{p}$ be the solution of $\Delta_{g} u_{1}=f\left(e_{1}\right)$ guaranteed by Theorem 2.8. Also let $\Delta v_{1}=f\left(e_{2}\right)$. Note since $8 \Delta\left(u_{1}-e_{1}\right)=8 \Delta\left(u_{1}\right)=R\left(e_{1}+1\right)$ $-M\left(e_{1}+1\right)^{-7} \leqq 0$ we have by the maximal principle that $u_{1} \geqq e_{1}>-1$. Similarly $v_{1} \leqq e_{2}$. It is clear there is a constant $C$ sufficiently large so that with $e_{1}<u<e_{2}$

$$
\frac{\partial f(x)}{\partial u}-C(R(x)+M(x)) \leqq 0 .
$$

Set $\lambda(x)=C(R+M)(x)$. Note that $\lambda \in M_{s-2, \delta+2^{\prime}}^{p}$ and $\lambda \geqq 0$. Let $T: M_{s, \delta}^{p} \rightarrow M_{s, \delta}^{p}$ be given by

$$
T(v)=\left(\Delta_{g}-\lambda\right)^{-1}(f(x)-\lambda v) .
$$

This makes sense since if $v \in M_{s, \delta}^{p}$ then $f(v)-\lambda v$ may be taken to be in $M_{s-2, \delta+2}^{p}$ and Theorem 2.8 applies. To see that $T$ is monotone let $w_{1} \leqq w_{2}$. Then since $f-\lambda$ is decreasing we find

$$
\left(\Delta_{g}-\lambda\right)\left(T\left(w_{1}\right)-T\left(w_{2}\right)\right)=(f-\lambda)\left(w_{1}\right)-(f-\lambda)\left(w_{2}\right) \geqq 0
$$


and so $T\left(w_{1}\right) \leqq T\left(w_{2}\right)$ by the maximum principle. Now letting $u_{i}=T\left(u_{i-1}\right)$ and $v_{i}=T\left(v_{i-1}\right)$ we get a pair of monotonic sequences of functions $-1<e_{1} \leqq u_{1} \leqq u_{2} \leqq u_{i}$ $\leqq \ldots \leqq v_{j} \leqq v_{j-1} \leqq \ldots \leqq e_{2}$ converging (at least) pointwise to $u(x) \leqq v(x)$. Note that both $u$ and $v$ are formally fixed points of $T$, i.e. using continuity of $T$

$$
T(u(x))=T\left(\lim _{i \rightarrow \infty} u_{i}(x)\right)=\lim _{i \rightarrow \infty} T\left(u_{i}(x)\right)=u(x) .
$$

Using the following and Step 1 we can conclude $u=v$ and $v$ is a solution to $\left(\mathrm{C}^{\prime \prime}\right)$.

Step 3. Regularity; $\left\{v_{i}\right\}$ converges in $M_{s, \delta}^{p}$.

First a note for each $i, v_{i} \sigma^{\delta} \in L^{p}$ thus using the monotone convergence theorem $v \sigma^{\delta} \in L^{p}$. Similarly $f(v) \sigma^{\delta+2} \in L^{p}$ since $f\left(v_{i}\right) \sigma^{\delta+2}$ is a monotonic sequence of functions in $L^{p}$. Now note for any solution $\phi$ to $\left(\mathrm{C}^{\prime \prime}\right)$ and any $\bar{s} \geqq 2$, there is a constant $B$

$$
|\phi|_{p, \bar{s}, \delta} \leqq B|\Delta \phi|_{p, \bar{s}-2, \delta+2}=B|f(\phi)|_{p, \bar{s}-2, \delta+2}
$$

and so letting $\bar{s}=2$ and $\phi=v$ we may conclude $v \in M_{2, \delta}^{p}$. Now $2>3 / p$ and so $f(v) \in M_{2, \delta}^{p}$. Thus we may make repeated use of (1) to establish $v \in M_{s, \delta}^{p}$.

Step 5. The neighborhood $U$.

Let $\phi$ be a solution to $\left(\mathrm{C}^{\prime \prime}\right)$ with given $g$ and $M$ and define a function $F$ :

$$
\begin{aligned}
& F: M_{s, \delta}^{p} \times \mathscr{R}_{s, \delta}^{p} \times M_{s-2, \delta+2}^{p} \rightarrow M_{s-2, \delta+2}^{p} \\
& F(\phi, g, M)=\Delta_{g} \phi-R(g) \phi+M(1+\phi)^{-7}-R(g) .
\end{aligned}
$$

Note $F(\phi, g, M)=0$. Also it follows from the coordinate formulas for $\Delta_{g}$ and $R(g)$ and Lemmas 2.4-2.6 that $F$ is $C^{\infty}$ at $(\phi, g, M)$. Also

$$
\frac{\partial F}{\partial \phi}(\phi, g, M): \psi \rightarrow \Delta_{g} \psi-R(g) \psi-7(1+\phi)^{-8} \psi
$$

and it follows from Theorem 2.8 and the maximal principle that $\frac{\partial F}{\partial \phi}(\phi, g, M)$ is an isomorphism from $M_{s, \delta}^{p}$ onto $M_{s-2, \delta+2}^{p}$. Thus it follows from the implicit function theorem there is a neighborhood $U$ of $(g, M)$ in $\mathscr{R}_{s, \delta}^{p} \times M_{s-2, \delta+2}^{p}$ and a unique function $\phi: U \rightarrow M_{s, \delta}^{p}$ such that $\phi(g, M)=\phi$ and $\mathrm{F}(\phi(g, M), g, M)=0$ for $(g, M) \in U$. Thus for $\left(g^{\prime}, M^{\prime}\right) \in U, \phi\left(g^{\prime}, M^{\prime}\right)$ is the desired solution. Q.E.D.

Since if $R=M=0, g=\gamma$, the Euclidean metric, then $\phi=1$ is a solution of $(E)$, Theorem 4.1 tells us we may solve (E) when $M$ is near 0 and $R$ has a small amount of negative curvature. This along with the remark that the solution of the constraint equation associated with $g$ only depends on $g$ 's conformal structure will allow us to find solution the constraint equations associated to members of $\mathscr{C}$ with non-positive curvature. This program is based on the following theorem:

Theorem 4.2. Let $g \in \mathscr{R}_{s, \delta}^{p}$ and $R(g) \leqq 0$. Let $\bar{R}$ be any function in $M_{s-2, \delta+2}^{p}$ such that $C_{1} \bar{R}(x) \leqq R(g)(x) \leqq \bar{R}(x) \leqq 0$ for some $C_{1} \geqq 0$. Then there is a unique positive $\phi \in M_{s, \delta}^{p}(1)$ (see Lemma 2.5) such that if $\bar{g}=\phi^{4} g$ the scalar curvature of $\bar{g}$ is $\bar{R}$. Furthermore, $\phi$ depends smoothly on $g$. 
Proof. It is well known that if $\bar{g}=\phi^{4} g$ and $R$ and $\bar{R}$ are the scalar curvatures of $g$ and $\bar{g}$ respectively then

$$
8 \Delta_{g}^{\phi}-R \phi+\bar{R} \phi^{5}=0 .
$$

Thus to prove this theorem we need show $(*)$ has a unique positive solution $\phi$ with $\phi-1 \in M_{s, \delta}^{p}$, and also that $\phi$ depends smoothly on $g$. We will break the proof into several steps:

Step 1 (Existence and uniqueness). We use a monotonic convergence scheme as in Theorem 4.1 and thus must establish existence of upper and lower solutions:

There is a constant upper solution $e_{1}>1$ iff $-R e_{1}+\bar{R} e_{1}^{5} \leqq 0$, i.e. if $\bar{R} e_{1}^{4} \leqq R$. Since $R$ and $\bar{R}$ are both negative and $c_{1} \bar{R} \leqq R$, such an $e_{1}$ clearly exists. A constant lower solution $e_{2}>0$ must satisfy $\bar{R} e_{2}^{4} \geqq R$. Since $\bar{R} \geqq R$ any $0<e_{2}<1$ will suffice. The existence proof proceeds exactly as in Theorem 4.1.

We may show uniqueness by picking $\lambda \in M_{s-2, \delta+2}^{p}$ such that $\lambda>0$ and such that $R u-\bar{R} u^{5}-\lambda u$ is decreasing in $u$. Solving (*) is equivalent to solving $8 \Delta_{g} u-\lambda u=R u$ $-\bar{R} u^{5}-\lambda u$ and thus the uniqueness argument of Theorem 4.1 applies.

We now show $\phi$ depends smoothly on $R$.

Step 2. Let $\frac{1}{5}<C<1$ and suppose $\bar{R} \leqq C R$. Then the solution $\phi$ found in Step 1 depends smoothly on $g$.

Proof. We use the implicit function theorem. Fixing $\bar{R}$ for the moment, we set

$$
F(g, \phi)=8 \Delta_{g} \phi-R_{g} \phi+\bar{R} \phi^{5},
$$

where $R_{g}$ is the scalar curvature of $g$. Since $\Delta_{g}$ and $R_{g}$ depend on at most two derivatives of $g$ and $s-2 \geqq 3 / p$ we see $F: \mathscr{C}_{s, \delta}^{p} \times M_{s, \delta}^{p}(1) \rightarrow M_{s-2, \delta+2}^{p}$ is smooth. Furthermore, a solution of $(*)$ is given by $F\left(g_{0}, \phi_{0}\right)=0$. Thus, we need show if $\left(g_{0}, \varphi_{0}\right)$ is a solution of $(\mathrm{E})$ then $\frac{F}{\partial \phi}\left(g_{0}, \phi_{0}\right): M_{s, \delta}^{p} \rightarrow M_{s-2, \delta+2}^{p}$ is a surjection with splitting kernal. In fact

$$
\frac{\partial F}{\partial \phi}\left(g_{0}, \phi_{0}\right)(v)=8 \Delta g_{0} v-R g_{0} v+\left(5 \bar{R} \phi_{0}^{4}\right) v
$$

is an isomorphism if $-R_{g_{0}}+5 \bar{R} \phi_{0}^{4} \leqq 0$. If $\left(g_{0}, \phi_{0}\right)$ satisfy the hypothesis of this step we have $5 \bar{R} \phi_{0}^{4} \leqq 5 C R_{g_{0}} \phi_{0}^{4}$ and so $5 \bar{R} \phi_{0}^{4} \leqq R_{g_{0}}$ if $5 C \phi_{0}^{4} \geqq 1$ (Recall $R_{g_{0}}<0$ ). Thus we need to show that $\phi_{0} \geqq\left(\frac{1}{5 C}\right)^{1 / 4}$. Since $C>\frac{1}{5}$ this reduces to showing $\phi_{0}>B$ for any $B<1$. An inspection of Step 1 shows such a $B$ is a lower solution of $(E)$ and the proof of this step is complete.

Step 3. The solution $\phi$ of $\left({ }^{*}\right)$ found is Step 1 depends smoothly on $g \in \mathscr{R}_{s, \delta}^{p}$.

Proof. Since $R$ is bounded we may choose a finite sequence of functions $R=R_{0} \leqq R_{1}$ $\leqq \ldots \leqq R_{n}=\bar{R}$ such that for each $i, 2 R_{i+1} \leqq R_{i}$. Applying Steps 1 and 2 we may for each $i$ find a unique positive $\phi_{i+1} \in M_{s, \delta}^{p}(1)$ satisfying

$$
8 \Delta_{g_{i}} \phi_{i+1}-R_{g_{i}} \phi_{i+1}+R_{i+1}\left(\phi_{i+1}\right)^{5}=0 \text {, }
$$


where $g_{i}=\phi_{1}^{4} \ldots \phi_{i}^{4} g$. Setting $\phi=\phi_{1} \ldots \phi_{n}$ and $\bar{g}=\phi^{4} g$ it is clear $\bar{g}$ is conformally equivalent to $g$. Also note that the fact $\phi$ does not depend on the choice of the functions $R_{i}$ follows from the uniqueness part of Step 1. Q.E.D.

We may combine the above results with those of the previous section to establish the following theorem:

Theorem 4.3. Let $(g, k) \in \mathscr{P}$. Suppose further that the scalar curvature $R$ of $g$ and $M=k \cdot k$ satisfy one of the following conditions.

1) There exist positive constants $C_{1}$ and $C_{2}$ such that $0 \leqq C_{1} R(x) \leqq M(x)$ $\leqq C_{2} R(x)$ for all $x \in \mathbb{R}^{3}$.

2) $R$ and $M$ are sufficiently near 0 in $M_{s-2, \delta+2}^{p}$.

3) $R \leqq 0$ and $M$ is sufficiently near 0 .

Then there is a unique positive function $\phi \in M_{s, \delta}^{p}(1)$ depending smoothly on $(g, k)$ such that the pair $\left(\phi^{4} g, \phi^{-2} k\right)$ satisfy the constraint equations.

Proof. For ( $g, k)$ satisfying Conditions 1$)$ and 2$)$ this is a restatement of Theorem 4.1. Note if $k \in S_{s-1, \delta+1}^{p}$ then a fortiori, $k \cdot k \in M_{s-2, \delta+2}^{p}$.

For $(g, k)$ satisfying Condition 3$)$ let $C>0$ be sufficiently small so that Condition 2) applies to $C R$. Let $\phi_{1}$ be the function guaranteed by Theorem 4.2 to give $g_{1}=\phi_{1}^{4} g$ scalar curvature $C R$. Applying Condition 2) gives us a factor $\phi_{2}$ such that $\bar{g}=\left(\phi_{1} \phi_{2}\right)^{4} g, \bar{k}=\left(\phi_{1} \phi_{2}\right)^{-2} k$ satisfy the constraint equations. Q.E.D.

Finally, since $s>3 / p$ and 1$), 2$ ), and 3 ) are open conditions in $C^{0}$, it follows the set of $(g, k)$ in $\mathscr{P}$ satisfying any one of the conditions is open.

\section{References}

1. Bourguignon,J.P., Ebin,D., Marsden,J.: Sur le noyau des operateurs pseudo-differential a symbole surjectif et non injectif. C. R. Acad. Sci. Paris A 282, 867- 870

2. Cantor, M. : Perfect fluid flows over $\mathbb{R}^{n}$ with asymptotic conditions. J. Func. Anal. 18, 73-84 (1975)

3. Cantor, M.: Some problems of global analysis on asymptotically simple manifolds. Comp. Math. (to appear)

4. Cantor, M.: Spaces of functions with asymptotic conditions. Indiana U. Math. J. 24, 397-902(1975)

5. Cantor, M., Fischer, A., Marsden,J., O'Murchadha, N., York, J.: The existence of maximal slicings in asymptotically flat spacetimes. Commun. math. Phys. 49, 187-190 (1976)

6. Choquet-Bruhat, Y.: Global solutions of the equations of constraints in general relativity on closed manifolds. Symp. Math. XIII, 317-325 (1973)

7. Choquet-Bruhat, Y.: Private correspondence

8. Choquet-Bruhat, Y.: Probleme des constraintes sur une variete compacte. C. R. Acad. Sci. Paris 274, $682-684(1972)$

9. Choquet-Bruhat, Y.: Sous-varietes, ou a courbure constante, de varietes lorentziennes. C. R. Acad. Sci. Paris 280, 169-171 (1975)

10. Choquet-Bruhat, Y., Marsden,J.: Solution of the local mass problem in general relativity. C. R. Acad. Sci. Paris 282, 609-612 (1976)

11. Fischer, A., Marsden, J. : The manifold of conformally equivalent metrics. Can. J. Math. 29, 193-209 (1977)

12. Kazden,J., Warner,F.: Scalar curvature and conformal deformation of Riemannian structure. J. Diff. Geom. 10, 113-134 (1975)

13. Lang, S. : Introduction to differentiable manifolds. New York: Interscience Publishers 1962

14. Lichnerowicz, A. : L'integration des equations de la gravitation probleme des $n$ corps. J. Math. Pures Appl. 23, 37-63 (1944)

15. Marsden,J.: Applications of global analysis in mathematical physics. Boston : Publish or Perish 1974 
16. Nirenberg, L., Walker, H.: The null space of elliptic partial differential operators in $\mathbb{R}^{n}$. J. Math. Anal. Appl. 47, 271-301 (1973)

17. O'Murchadha, N., York, J.: Existence and uniqueness of solutions of the Hamiltonian constraint of general relativity on compact manifolds. J. Math. Phys. 14, 1551-1557 (1973)

18. O’Murchadha, N., York,J. : Initial-value problem of general relativity. I. General formulation and physical interpretation. Phys. Rev. D10, 428 - 436 (1974)

19. Protter, M., Weinberger,H.: Maximal principles in differential equations. Englewood Cliffs, NJ : Prentice Hall 1967

20. York, J.W.: Conformally invariant orthogonal decomposition of symmetric tensors on Riemannian manifolds and the initial value problem of general relativity. J. Math. Phys. 14, 456-464 (1973)

21. York,J.W.: Covariant decomposition of symmetric tensors in the theory of gravitation. Ann. Inst. H. Poincare 21, 319-332 (1974)

Communicated by R. Geroch

Received June 7, 1977

\section{Note Added in Proof}

The proof of Step 2 of Theorem 4.1 should be modified as follows :

We may choose $e_{1}<0$ and $e_{2}>0$ so that for $f(x, u)=8^{-1}\left(R(x)(u+1)-M(x)(u+1)^{-7}\right.$ we have $f\left(x, e_{1}\right) \leqq 0$ and $f\left(x, e_{2}\right) \geqq 0$. For $T$ as in the proof in the paper let $u_{1}=T\left(e_{1}\right)$ and $v_{1}=T\left(e_{2}\right)$. We claim $e_{1} \leqq u_{1}$ and $v_{1} \leqq e_{2}$. To see this note

$$
\begin{aligned}
\left(\Delta_{g}-\lambda\right)\left(u_{1}-e_{1}\right) & =\left(\Delta_{g}-\lambda\right) u_{1}-\left(\Delta_{g}-\lambda\right) e_{1} \\
& =f\left(x, e_{1}\right)-\lambda e_{1}-\Delta_{g}\left(e_{1}\right)+\lambda\left(e_{1}\right) \\
& =f\left(x, e_{1}\right) \leqq 0 .
\end{aligned}
$$

Also note $u_{1}(x)-e_{1} \rightarrow-e_{1} \geqq 0$ as $x \rightarrow \infty$ thus by the maximal principle

$u_{1}(x)-e_{1} \geqq-e_{1} \geqq 0$ for all $x$.

The proof that $v_{1} \leqq e_{2}$ is similar. Since $T$ is monotone and $e_{1} \leqq e_{2}$ it is clear that $u_{1} \leqq v_{1}$ and in fact if $u_{n}$ $=T\left(u_{n-1}\right)$ and $v_{n}=T\left(v_{n-1}\right)$ that $u_{n} \leqq v_{n}$ for all $n$. The proof proceeds as in the paper. 